\title{
Polarization-singular processing of biological layers laser images to diagnose and classify their optical properties
}

\author{
Yu.O. Ushenko*a, O.Yu. Telenga ${ }^{\mathrm{b}}$ \\ ${ }^{a}$ Correlation Optics Department, Chernivtsi National University, 2 Kotsyubinsky Str., 58012, \\ Chernivtsi, Ukraine; \\ ${ }^{\mathrm{b}}$ Optics and Spectroscopy Department, Chernivtsi National University, 2 Kotsyubinsky Str., 58012, \\ Chernivtsi, Ukraine;
}

\begin{abstract}
Presented in this work are the results of investigation aimed at analysis of coordinate distributions for azimuths and ellipticity of polarization (polarization maps) in blood plasma layers laser images of three groups of patients: healthy (group 1), with dysplasia (group 2) and cancer of cervix uteri (group 3). To characterize polarization maps for all groups of samples, the authors have offered to use three groups of parameters: statistical moments of the first to the fourth orders, autocorrelation functions, logarithmic dependences for power spectra related to distributions of azimuths and ellipticity of polarization inherent to blood plasma laser images. Ascertained are the criteria for diagnostics and differentiation of cervix uteri pathological changes.
\end{abstract}

Keywords: polarization, singularity, laser image, blood plasma, statistic moment, autocorrelation, fractal, dysplasia, cancer

\section{INTRODUCTION}

By tradition, the processes of transforming optical radiation of phase-inhomogeneous objects and media are considered, as a rule, in a statistical approach (theory of radiation $\operatorname{transfer}^{1}$, Monte-Carlo modeling ${ }^{2}$ ). Among the most spread traditional methods for studying the scattered light fields, one can separate the following independent directions: "scalar" (photometry and spectrophotometry) ${ }^{3,4}$ and "vector" (polarization nephelometry, Mueller-matrix optics) $)^{5}$. Using these approaches, determined are interrelations between the sets of statistical moments of the 1-st to the 4-th orders ${ }^{5-7}$, correlation functions ${ }^{7}$, fractal dimensions ${ }^{8}$ that characterize phase-inhomogeneous biological layers and coordinate distributions for phases, azimuths and ellipticity of polarization in their laser images.

In parallel with traditional statistical investigations, formed in recent 10 to 15 years is the new optical approach to describe a structure of polarizationally inhomogeneous fields in the case of scattered coherent radiation. The main feature of this approach is the analysis of definite polarization states to determine the whole structure of coordinate distributions for azimuths and ellipticities of polarization. The so-called polarization singularities are commonly used as these states ${ }^{7}$ :

states with linear polarization when the direction of rotation for the electric field vector is indefinite, the so-called L-points;

circularly-polarized states when the azimuth of polarization for the electric field vector is indefinite, the so-called C-points.

This work is aimed at ascertaining the possibilities to diagnose and classify of cervix uteri pathological changes by determination values and ranges for changing the statistical (moments of the 1-st to the 4-th orders), correlation (autocorrelation functions) and fractal (logarithmic dependences for power spectra) parameters that characterize coordinate distributions for polarization-singular states in blood plasma (BP) laser images.

*yuriyu@gmail.com

Tenth International Conference on Correlation Optics, edited by Oleg V. Angelsky, Proc. of SPIE Vol. 8338, 83380U · (c) 2011 SPIE · CCC code: 0277-786X/11/\$18 - doi: 10.1117/12.920074 


\section{POLARIZATION MODELING OF PROPERTIES INHERENT TO NETWORKS OF BIOLOGICAL PROTEIN CRYSTALS IN BLOOD PLASMA}

As a base for analyses of processes providing formation of polarization-inhomogeneous images of blood plasma, we use the optical model developed in ${ }^{5-8}$ :

- $\quad$ optical properties of blood plasma are determined as those of a two-component amorphous-crystalline structure;

- $\quad$ crystalline component is an architectonic net consisting of amino-acid liquid crystals;

- $\quad$ optically, the amino-acid liquid crystals possess the properties of uniaxial birefringent crystals.

The optical properties of amorphous $\{A\}$ and crystalline $\{C\}$ components of blood plasma can be exhaustively described using the following Jones operators ${ }^{5}$

$$
\begin{gathered}
\{A\}=\left\|\begin{array}{ll}
a_{11} & a_{12} \\
a_{21} & a_{22}
\end{array}\right\|=\left\|\begin{array}{cc}
\exp (-\tau) & 0 \\
0 & \exp (-\tau l) \|
\end{array}\right\| \\
\{C\}=\left\|\begin{array}{ll}
c_{11} & c_{12} \\
c_{21} & c_{22}
\end{array}\right\|=\| \begin{array}{ll}
\cos ^{2} \rho+\sin ^{2} \rho \exp (-i \delta) ; & \cos \rho \sin \rho[1-\exp (-i \delta)] ; \\
\cos \rho \sin \rho[1-\exp (-i \delta)] ; & \sin ^{2} \rho+\cos ^{2} \rho \exp (-i \delta) ; \|
\end{array} .
\end{gathered}
$$

Here, $\tau$ is the absorption coefficient for laser radiation in the blood plasma layer with the geometric thickness $l ; \rho$ direction of the optical axis; $\delta=2 \pi / \lambda \Delta n d$ - phase shift between the orthogonal components $E_{x}$ and $E_{y}$ of the amplitude of illuminating laser light with the wavelength $\lambda ; \Delta n$ - index of birefringence.

The Jones matrix of the blood plasma layer, where isotropic and anisotropic creations lie in one plane, can be expressed as a sum of operators $\{A\}$ and $\{C\}$

$$
\{M\}=\{A\}+\{C\}=\left\|\begin{array}{ll}
a_{11}+c_{11} ; & a_{12}+c_{12} ; \\
a_{21}+c_{21} ; & a_{22}+c_{22}
\end{array}\right\|,
$$

If taking into account the relations (1) - (3) it can be shown that polarization-inhomogeneous laser image of blood plasma with the following values of azimuth $\alpha(r)$ and ellipticity $\beta(r)$ is formed in every point $(r)$.

$$
\left\{\begin{array}{l}
\alpha(r)=\arccos \left(\sin \delta / \cos 2\left(\operatorname{arctg}\left\{\left[\left(m_{21}+m_{22}\right)^{2} /\left(m_{11}+m_{12}\right)^{2}\right]\right\}\right)\right. \\
\beta(r)=\arcsin \left(\operatorname{tg} \delta / \sin 2\left(\operatorname{arctg}\left\{\left[\left(m_{21}+m_{22}\right)^{2} /\left(m_{11}+m_{12}\right)^{2}\right]\right\}\right)\right.
\end{array}\right.
$$

As it follows from the analytical relations (4) among the whole set of values $(\alpha(r) ; \beta(r))$, formation of $L$ and $\pm C$ polarization states seems to be very probable

$$
" L-" \Leftrightarrow \delta(r)=q \pi, \quad q=0 ; 1 ; 2 ; \ldots
$$




$$
\pm C-\Leftrightarrow \operatorname{tg} \delta(r)=\sin 2\left(\operatorname{arctg}\left\{\left[\left(m_{21}(r)+m_{22}(r)\right)^{2} /\left(m_{11}(r)+m_{12}(r)\right)^{2}\right]\right\}\right) .
$$

In this work, to describe coordinate distributions for polarization-singular $(L, \pm C$ ) states in laser images for all the types of $\mathrm{BP}^{5}$ we have used the following characteristic values of the fourth Stokes vector parameter $\left(V_{4}\right)$ in corresponding points $(x, y)$

$$
\left\{\begin{array}{l}
V_{4}(x, y)=0 \leftrightarrow L(\beta=0) \\
V_{4}(x, y)= \pm 1 \leftrightarrow \pm C(\beta= \pm \pi / 4)
\end{array}\right.
$$

\section{EXPERIMENTAL SETUP FOR POLARIMETRIC INVESTIGATIONS}

Our study of polarization-inhomogeneous laser images inherent to BP was performed using the optical scheme of a laser polarimeter (Fig. 1) $)^{5-7}$
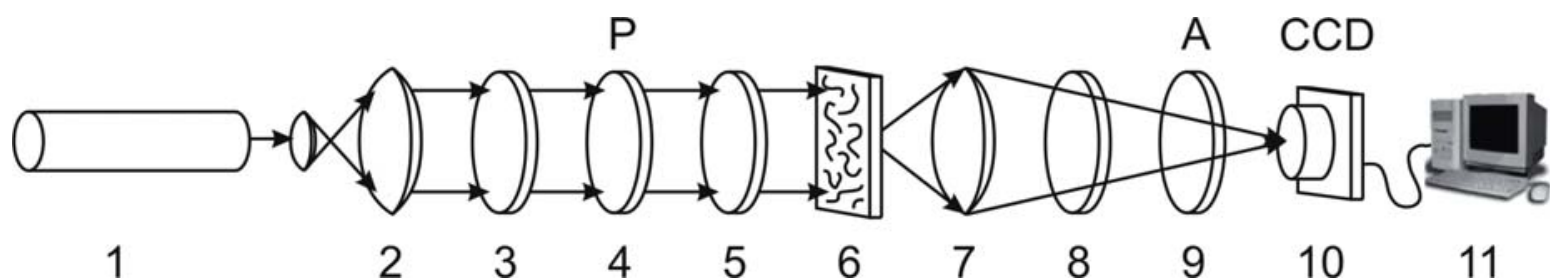

Figure 1. Optical scheme of the polarimeter: 1 - He-Ne laser; 2 - collimator; 3, 5, 8 - quarter-wave plates; 4, 9 - polarizer and analyzer, respectively; 6 - object under investigation; 7 - micro-objective; 10 - CCD camera; 11 - personal computer.

Illumination was performed using a parallel beam $\left(\varnothing=10^{4} \mu \mathrm{m}\right)$ from a He-Ne laser $(\lambda=0.6328 \mu \mathrm{m}) 1$. The polarization illuminator (quarter-wave plates 3 and 5 as well as polarizer 4) were used to form various polarization states in the laser beam. Polarization images of BP 6 were projected using the micro-objective 7 into the plane of the light-sensitive area ( $m \times n=800$ pix $\times 600$ pix $)$ in CCD camera 10. Turning the transmission axis of the analyzer 9 by the angles $\pm 45^{0}$ relatively to the direction of the highest velocity axis for the quarter-wave plate 8 , wee could determine the intensities of right $\left(I_{\otimes}\right)$ and left $\left(I_{\oplus}\right)$ circularly polarized components for each separated pixel of CCD camera 10. It served as a base to calculate coordinate distributions of the fourth parameter in the Stokes vector $\left.V_{4}(m \times n)\right)$ describing the laser image of $\mathrm{BP}$, if using the relation

$$
V_{4}\left(r_{m n}\right)=\frac{I_{\otimes}\left(r_{i k}\right)-I_{\oplus}\left(r_{i k}\right)}{I_{\otimes}\left(r_{i k}\right)+I_{\oplus}\left(r_{i k}\right)} .
$$

The two-dimensional array (7) was scanned along the horizontal direction $x \equiv 1, \ldots, m$ with the step $\Delta x=1$ pix . Within the limits of each local sample $\left.\left(1_{p i x} \times n_{p i x}\right)^{(k=1,2}, \ldots, m\right)$, we calculated the amount $(N)$ of characteristic values $V_{4}(k)=0,-\left(N_{L}^{(k)}\right)$ and $V_{4}(k)= \pm 1,-\left(N_{ \pm C}^{(k)}\right)$.

Thus, we determined the dependences $N_{L}(x) \equiv\left(N_{L}{ }^{(1)}, N_{L}^{(2)}, \ldots, N_{L}^{(m)}\right) \quad$ and $N_{ \pm C}(x) \equiv\left(N_{ \pm C}{ }^{(1)}, \quad N_{ \pm C}{ }^{(2)}, \ldots, \quad N_{ \pm C}{ }^{(m)}\right)$ for amounts of polarization-singular $L-$ and $\pm C$ - points within the limits of a laser image for BP. 


\section{CRITERIA TO ESTIMATE POLARIZATION-INHOMOGENEOUS IMAGES OF BP}

Distributions $N_{L ; \pm C}(x)$ for the amount of polarization-singular states in laser images of BP are characterized with the set of statistical moments of the 1-st to the 4-th orders $Z_{j=1 ; 2 ; 3 ; 4}$ calculated using the following relations

$$
\begin{gathered}
Z_{1}=\frac{1}{M} \sum_{i=1}^{M} \mid N_{L, \pm C}^{(i)}(x), \quad Z_{2}=\sqrt{\frac{1}{M} \sum_{i=1}^{M}\left[N_{L, \pm C}^{(i)}(x)\right]^{2}}, \\
Z_{1}=\frac{1}{Z_{2}^{3}} \frac{1}{M} \sum_{i=1}^{M}\left[N_{L, \pm C}^{(i)}(x)\right]^{2}, \quad Z_{4}=\frac{1}{Z_{2}^{4}} \frac{1}{M} \sum_{i=1}^{M}\left[N_{L, \pm C}^{(i)}(x)\right]^{4} .
\end{gathered}
$$

where $M=800 \times 600$ is the amount of pixels in CCD camera 10 (Fig. 1).

Our analysis of the coordinate structure for $N_{L ; \pm C}(x)$ distributions was based on the autocorrelation method by using the function ${ }^{5}$

$$
K_{L, \pm C}(m)=\frac{1}{(n-m) \sigma^{2}} \sum_{t=1}^{n-m}\left[X_{t}-\mu\right]\left[X_{t+m}-\mu\right] .
$$

Here, $n$ is the length of discrete sampling $N_{L, \pm C}(x)=X_{1}, X_{2}, \ldots, X_{n} ; \mu$ - average value, $\sigma^{2}$ - the dispersion; $m, n$ - positive integers; $(m=1$ pix $)$ is the step for changing the coordinate $x=1 \div m$.

As correlation parameters that characterize the dependences $K_{L ; \pm C}(\Delta x)$, we chose:

- correlation area $S_{L ; \pm C}$

$$
S_{L ; \pm C}=\int_{1}^{m} K_{L ; \pm C}(m) d m ;
$$

- correlation moment $Q_{L ; \pm c}$ that define the kurtosis for the distribution of values $K_{L ; \pm C}(\Delta x)$

$$
Q_{L, \pm C}=\frac{N}{\left(\sum_{i=1}^{N}\left(K_{L, \pm C}(m)\right)_{i}^{2}\right)^{2}} \sum_{i=1}^{N}\left(K_{L, \pm C}(m)\right)_{i}^{4}
$$

The fractal analysis of the distributions $N_{L ; \pm C}(x)$ was performed using the calculation of logarithmic dependences $\log J\left[N_{L ; \pm C}(x)\right]-\log d^{-1}$ for the power spectra $J\left[N_{L ; \pm C}(x)\right]$, which was calculated as a discrete Fourier transform of the corresponding autocorrelation function $K_{L, \pm C}(m)$ using the MatLab software

$$
J\left[N_{L, \pm C}(x)\right]=S_{x x}(w)=\sum_{m=1}^{n} K(m) e^{-j \omega m},
$$

where $\omega=d^{-1}$ are the spatial frequencies that are determined by geometrical sizes $(d)$ of BP structural elements.

The dependences $\log J\left[N_{L ; \pm C}(x)\right]-\log d^{-1}$ are approximated using the least-squares method into the curves $\Phi(\eta)$, straight parts of which serve to determine the slope angles $\eta$ and calculate fractal $F$ dimensionalities by using the relations ${ }^{6}$

$$
F_{L ; \pm C}=3-\operatorname{tg} \eta
$$

Classification of coordinate distributions $N_{L ; \pm C}(x)$ should be performed using the following criteria:

- $\quad$ they are fractal on the condition of a constant slope angle value $\eta=$ const for 2 to 3 decades of changing sizes $d$; 
- $\quad$ they are multi-fractal, if several slope angles $\Phi(\eta)$ are available;

- $\quad$ they are random (statistic) when any stable slope angles are absent within the whole range of changing sizes $d$.

In the latter case, the distributions $\log J\left[N_{L ; \pm C}(x)\right]-\log d^{-1}$ are characterized with the dispersion

$$
D_{z}=\sqrt{\frac{1}{m} \sum_{i=1}^{m}\left[\log J\left(N_{L ; \pm C}\left(x_{i}\right)\right)-\log d^{-1}\right]^{2}} .
$$

\section{CHARACTERIZATION OF OBJECTS UNDER INVESTIGATION}

Fig. 2 illustrates coordinate $(100$ pix $\times 50$ pix $)$ distributions of the fourth parameter for the Stokes vector $V_{4}(m \times n)$ inherent to laser images of BP in all the groups.

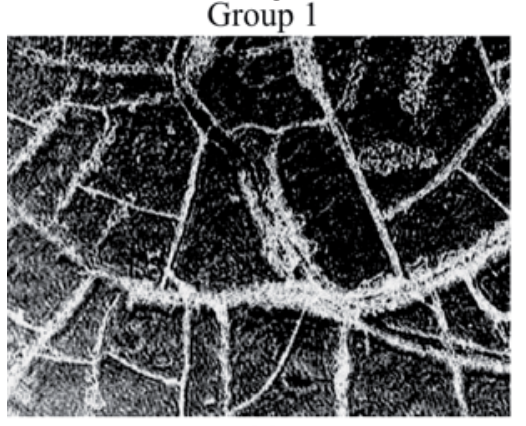

(a)

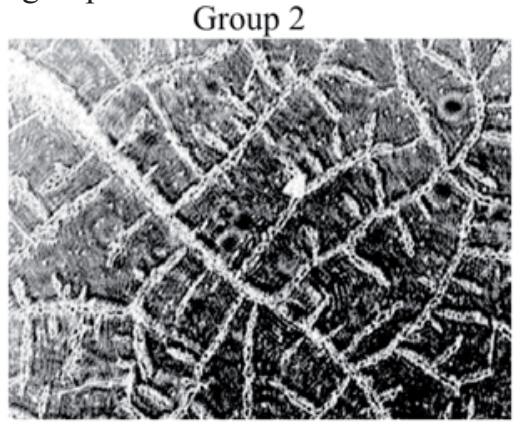

(b)

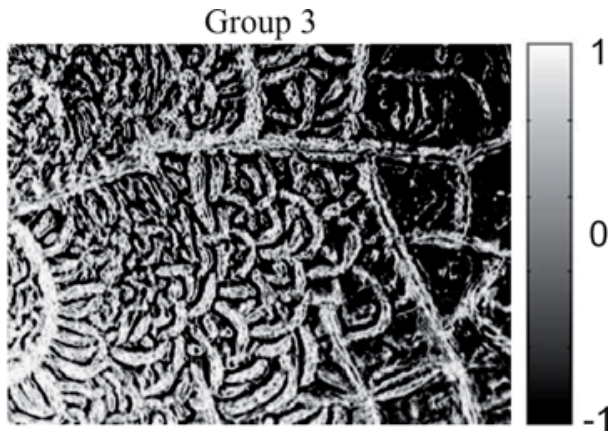

(c)

Figure 2. Coordinate distributions of the fourth Stokes vector parameter $V_{4}(m \times n)$ of laser images inherent to BP.

Our qualitative analysis of coordinate distributions $V_{4}(m \times n)$ for laser images of BP (Fig. 2) enabled to reveal:

- the image of BP layer of a healthy patient (Fig. 2a) contains of elliptically polarized areas of protein crystals network $\left(V_{4}(m \times n) \neq 0\right)$ on a linear polarized background $V_{4}(m \times n)=0$;

- the image of BP layer of a patient with mastopathy shows the increasing of polarization inhomogeneous component (Fig. 2b) formed both by linearly $\left(V_{4}(m \times n)=0\right)$ and elliptically $\left(V_{4}(m \times n) \neq 0\right)$ polarized states, including the circularly $\left(V_{4}(m \times n)=1\right)$ polarized one;

- the image of BP layer of a patient with cervix uteri cancer changes is characterized with the widest range of changing the azimuth and ellipticity of polarization, which is connected with developed polycrystalline network (Fig. 2c) of amino acids of albumin and globulin $\left(-1 \leq V_{4}(m \times n) \leq 1\right)$.

\section{STATISTICAL, CORRELATION AND FRACTAL ANALYSIS FOR DISTRIBUTIONS OF POLARIZATION-SINGULAR STATES IN LASER IMAGES OF BP}

\section{1. $L$ - states of laser images}

Summarized in Fig. 3 is a series of coordinate $\left(V_{4}(m \times n)=0\right.$, - fragments (a), (b), (c)), quantitative $\left(N_{L}(x)\right.$, fragments (d), (e), (f)), autocorrelation $\left(K_{L}(\Delta m)\right.$, - fragments $\left.(\mathrm{g}),(\mathrm{h}),(\mathrm{k})\right)$ and $\log \operatorname{arithmic}\left(\log J_{L}-\log d^{-1}\right.$, fragments (l), (m), (n)) distributions for polarization-singular L states in all groups blood plasma layers laser images.

Our comparative analysis of the obtained dependences $N_{L}(x)$ for the amount of polarization-singular L - states in laser images of BP in all the groups (Figs 3 (d) - (n)) revealed similarity of their statistical, correlation and fractal structures.

For instance, all the $N_{L}(x)$ distributions are close to the equiprobable ones - the condition $Z_{j=3 ; 4}^{L}<<Z_{j=1 ; 2}^{L}$ is valid for the values of statistical moments (relations (8)). Distinctions between the distributions of L - states in laser images of 
various $\mathrm{BP}$ are observed as variations of the 1-st and the 2-nd statistical moments $Z_{1}^{L}=0.59 ; Z_{2}^{L}=0.15 ; Z_{3}^{L}=0.06 ; Z_{4}^{L}=0.08 \quad($ group 1$) ; \quad Z_{1}^{L}=0.44 ; Z_{2}^{L}=0.22 ; Z_{3}^{L}=0.08 ; Z_{4}^{L}=0.088$ (group 2) and $Z_{1}^{L}=0.29, Z_{2}^{L}=0.36 ; Z_{3}^{L}=0.05 ; Z_{4}^{L}=0.06$ (group 3).

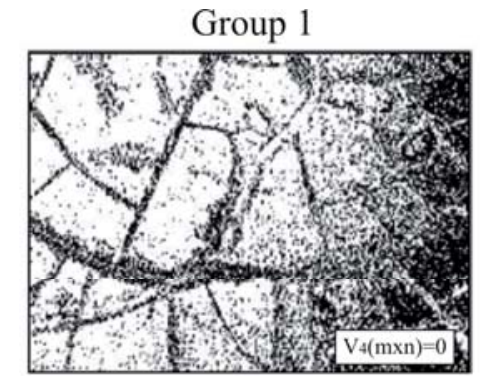

(a)
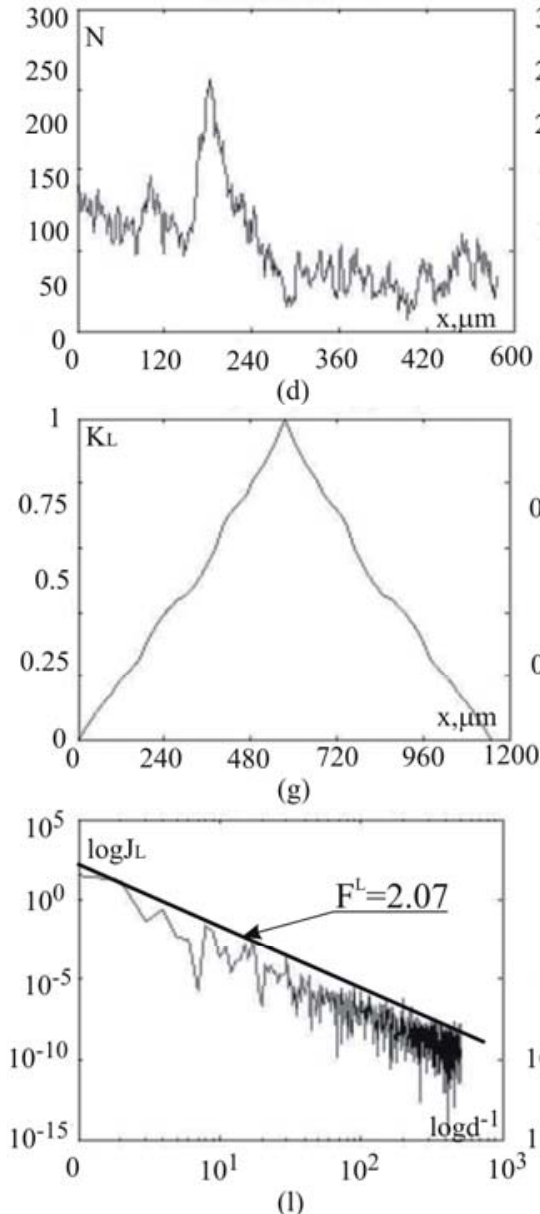

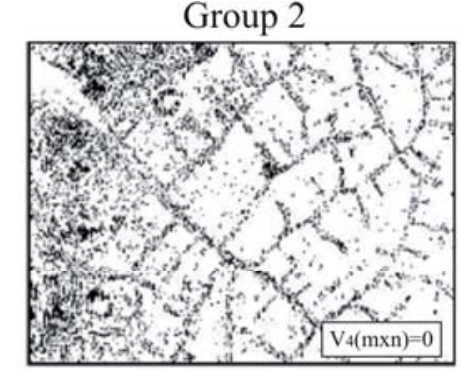

(b)

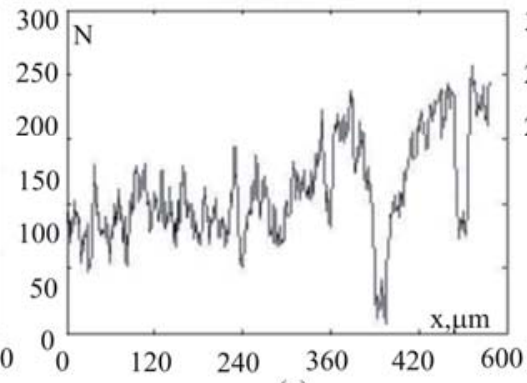

(e)
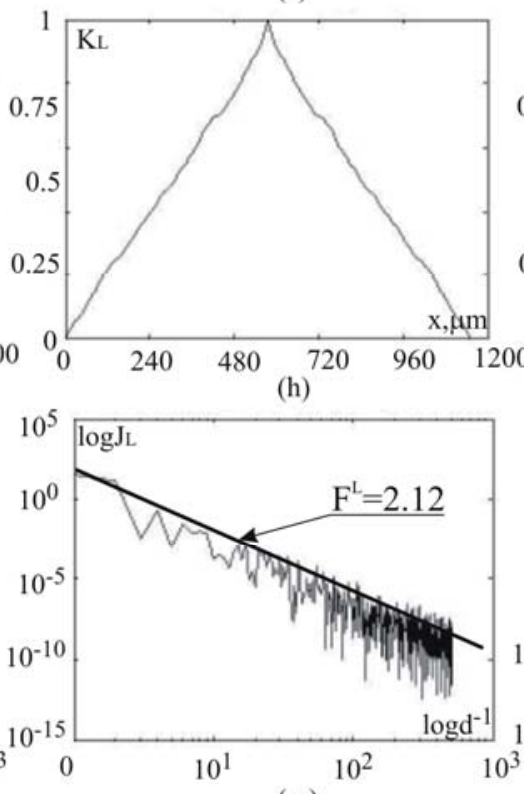

$(\mathrm{m})$

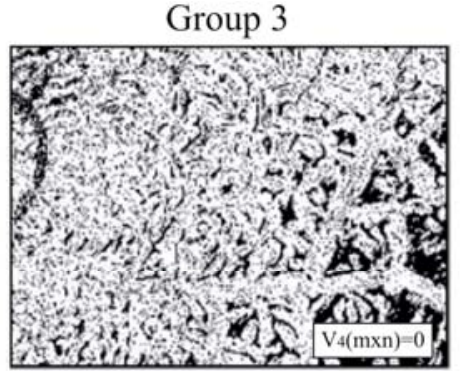

(c)

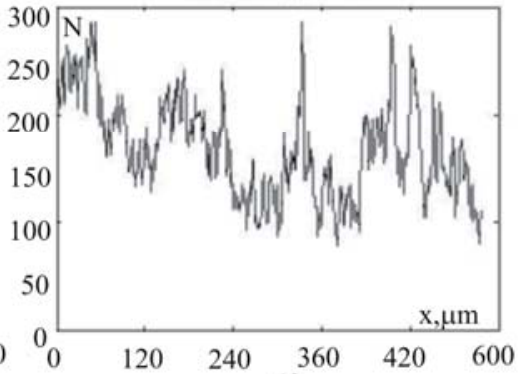

(f)

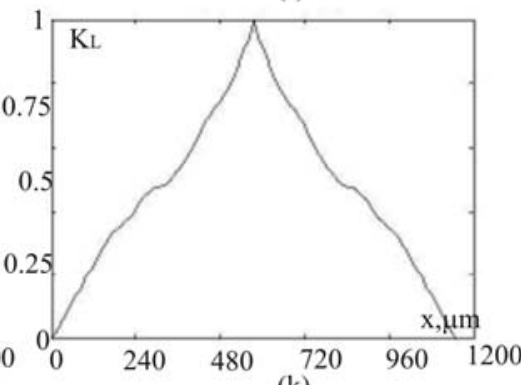

(k)

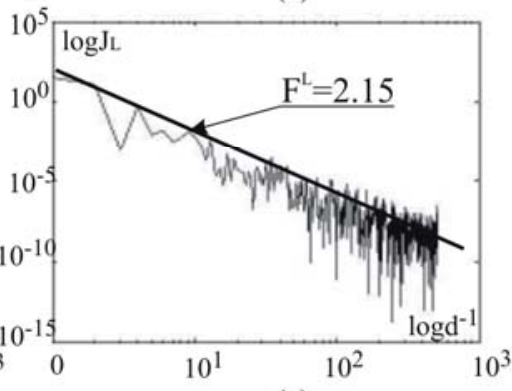

(n)

Figure 3. Coordinate $V_{4}(m \times n)=0$ ((a), (b), (c)) and quantitative $N_{L}(x)$ distributions ((d), (e), (f)) of L - states in polarization; autocorrelation functions $K_{L}(\Delta m)((\mathrm{g}),(\mathrm{h}),(\mathrm{k}))$ and dependencies $\log J_{L}-\log d^{-1}((\mathrm{l}),(\mathrm{m})$, (n)) for power spectra $J\left(N_{L}\right)$ of the distribution $N_{L}(x)$ for laser images of the BP layers.

As seen, for BP of the 1-st, 2-nd and 3-rd groups, the mean value $Z_{1}^{L}$ is 1.5 and 2.1 times decreased. In addition, vice versa, the dispersion $Z_{2}^{L}$ is 1.5 and 2.45 times increased. These changes in $N_{L}(x)$ distributions are related with the 
mechanisms of optical anisotropy increasing (formation of elliptically polarized states in laser images), which are caused by increasing of BP proteins concentration.

The investigated statistical structure of L - states for polarization of BP layers laser images is confirmed by a monotonous drop of dependences for autocorrelation functions $K_{L}(\Delta m)$ (relations (9)) of all the distributions $N_{L}(x)$ (Figs. 3(g), (h), (k)). In this case, values of the correlation area $S$ and correlation moment $Q$ trend to their boundary values $\left(S^{L} \rightarrow 0,33 ; Q^{L} \rightarrow 0\right.$, relations (10) and (11)) that are characteristic just to equiprobable distributions: $S^{L}=0,24 ; Q^{L}=0,12$ (group 1); $S^{L}=0,22 ; Q^{L}=0,14$ (group 2) i $S^{L}=0,18 ; Q^{L}=0,19$ (group 3).

The performed analysis of logarithmic dependences $\log J_{L}-\log d^{-1}$ (Figs. 3(1), (m), (n)) for the power spectra $J\left(N_{L}\right)$ (relation (12) of the distribution $N_{L}(x)$ inherent to laser images of the group 1, group 2 and group 3 revealed a common regularity - the approximating curves are characterized with stable slope angles that are corresponded with increasing by their value fractal dimensionalities (relation (13)): $F^{L}=2.07$ (group 1); $F^{L}=2.12$ (group 2) and $F^{L}=2.15$ (group 3).

6.2. $\pm \mathbf{C}$ states of laser images

Summarized in Fig. 4 is the series of coordinate $\left(V_{4}(m \times n)=1\right)$, quantitative $\left(N_{ \pm C}(x)\right)$, autocorrelation $\left(K_{ \pm C}(\Delta m)\right)$ and logarithmic $\left(\log J_{ \pm C}-\log d^{-1}\right)$ distributions for polarization-singular $\pm C$-states in laser images of BP layers.

Optical manifestations of the anisotropic layer of BP protein network are illustrated with the network of $\pm C$ - points in the laser image (Fig. 4a, b, c). It is seen that the total amount of circularly polarized points in BP laser images of patients with pathological changes (Fig. $4 \mathrm{~d}, \mathrm{e}, \mathrm{f}$ ) is sequentially increasing. All the statistic moments $Z_{j=1 ; 2 ; 3 ; 4}^{ \pm C}$ that characterize the distribution $N_{ \pm C}(x)$ of the amount of circularly polarized singular states differ from zero: $Z_{1}^{ \pm C}=0.19 ; Z_{2}^{ \pm C}=0.31 ; Z_{3}^{ \pm C}=0.44 ; Z_{4}^{ \pm C}=0.58$ (group 1); $Z_{1}^{ \pm C}=0.29 ; Z_{2}^{ \pm C}=0.23 ; Z_{3}^{ \pm C}=0.87 ; Z_{4}^{ \pm C}=0.95$ (group 2) and $Z_{1}^{ \pm C}=0.34 ; Z_{2}^{ \pm C}=0.17 ; Z_{3}^{ \pm C}=1.54 ; Z_{4}^{ \pm C}=2.15$ (group 3). As seen, for BP of the 1-st, 2-nd and 3-rd groups, the values $Z_{3}^{ \pm C}, Z_{4}^{ \pm C}$ is $2-3,3$ times 1.85 and 3.84 is times decreased.

For the BP laser image of patients with mastopathy the correlation area $S^{ \pm C}$ and correlation moment $Q^{ \pm C}$ of the distribution $N_{ \pm C}(x)$ as compared with similar correlation parameters of $N_{L}(x)$ distributions (Fig. 3e) experience changes in inverse proportion: $S^{ \pm C}(\downarrow)=0.17$ and $Q^{ \pm C}(\uparrow)=0.59$.

The total amount of L- and $\pm C$ - states of polarization for the respective BP laser image of patients with cancer changes is approximately the same (Figs 3f, 4f). Like to the case of $N_{L}(x)$ distribution for the amount of linearly polarized states, the $N_{ \pm C}(x)$ distribution is close to the equiprobable one: $Z_{j=3 ; 4}^{ \pm C}<<Z_{j=1 ; 2}^{ \pm C}$. Differences between the statistical moments $Z_{j=1 ; 2 ; 3 ; 4}^{L}$ and $Z_{j=1 ; 2 ; 3 ; 4}^{ \pm C}$ are insignificant and lie within 20 to $30 \%$ : $Z_{1}^{ \pm C}=0.15 ; Z_{2}^{ \pm C}=0.32 ; Z_{3}^{ \pm C}=0.09 ; Z_{4}^{ \pm C}=0.17$.

The values of the correlation area $S^{ \pm C}$ and correlation moment $Q^{ \pm C}$ are close to their extremum ones: $S^{ \pm C}=0.23 ; Q^{ \pm C}=0.12$.

Our analysis of the logarithmic dependences (Fig. 4n) for the power spectra $J\left(N_{ \pm C}\right)$ of the $N_{ \pm C}(x)$ distribution in laser images of the BP layers of group 3 found a stable slope of the approximating curve: $F^{ \pm C}=2.11$.

The statistically averaged (within the limits of groups 1 to 3 ) values and ranges of changing statistical moments $Z_{j=1 ; 2 ; 3 ; 4}^{L ; \pm C}$, correlation $Q^{L ; \pm C}, S^{L ; \pm C}$ and fractal $F^{L ; \pm C}, D^{L ; \pm C}$ parameters that characterize the $N_{ \pm C}(x)$ dependences for the amount of singular states in laser images of BP have been illustrated on Table 1. 


\section{Group 1}

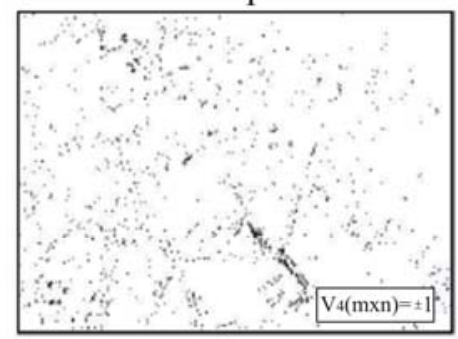

(a)

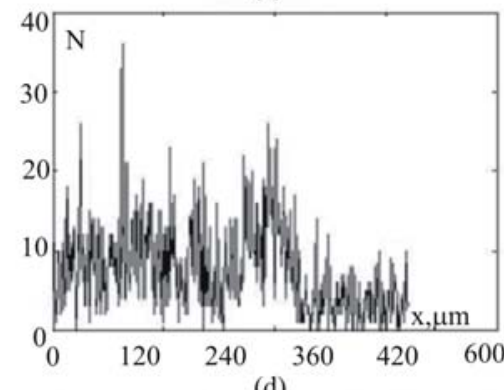

(d)

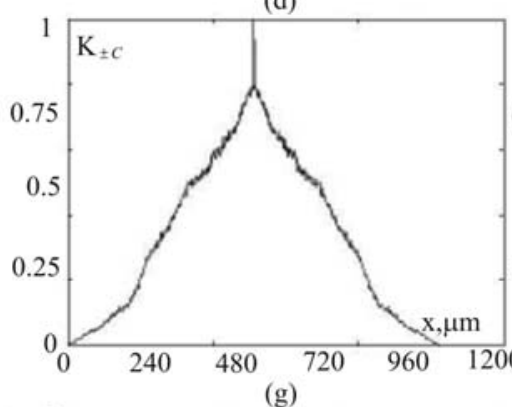

(g)

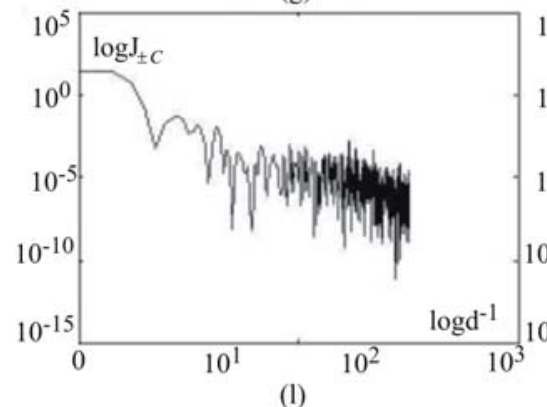

(l)
Group 2

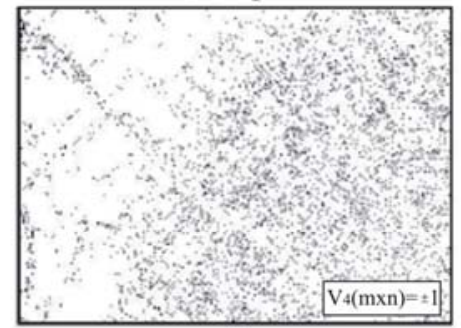

(b)
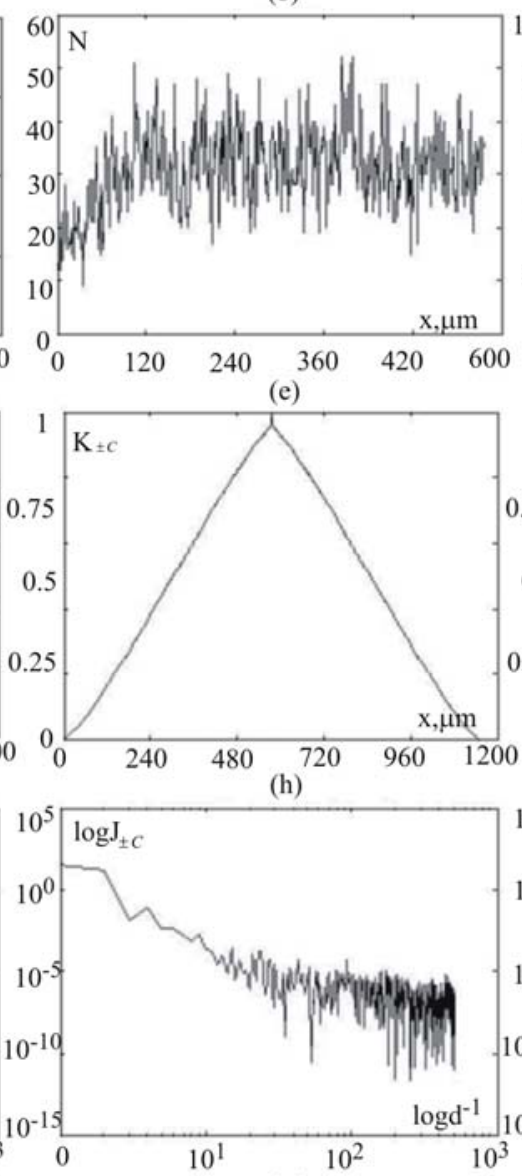

(m)
Group 3

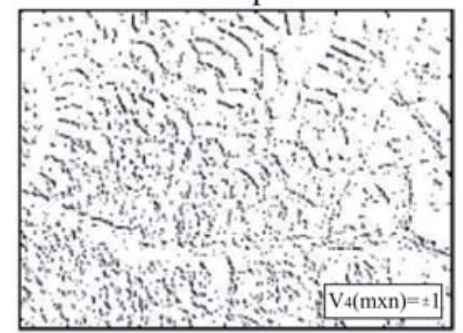

(c)

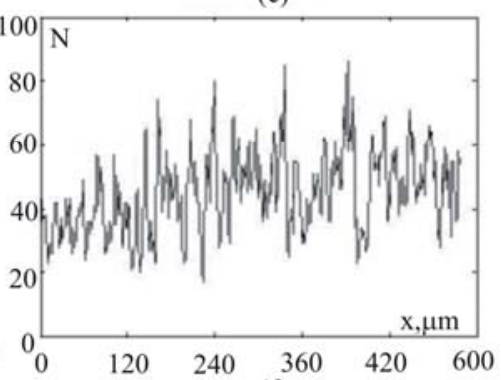

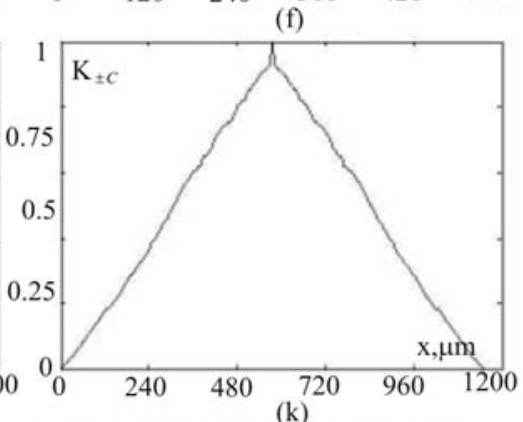

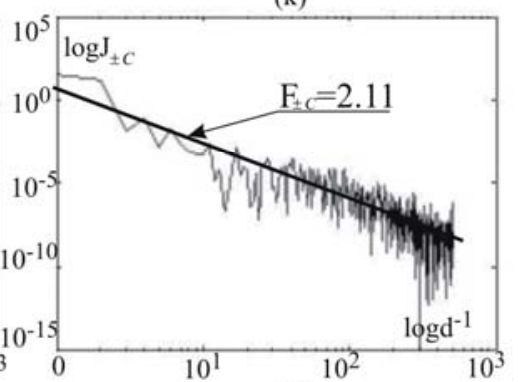

(n)

Figure 4. Coordinate $V_{4}(m \times n)=1$ ((a), (b), (c)), and quantitative $N_{ \pm C}(x)$ distributions ((d), (e), (f)), of $\pm C-$ states in polarization; autocorrelation functions $K_{ \pm C}(\Delta m)((\mathrm{g}),(\mathrm{h}),(\mathrm{k}))$ and dependencies $\log J_{L}-\log d^{-1}((\mathrm{l}),(\mathrm{m})$, (n)) for power spectra $J\left(N_{ \pm C}\right)$ of the distribution $N_{ \pm C}(x)$ for laser images of BP layers all groups. 
Table 1. Statistical, correlation and fractal parameters for the distribution of the amount of polarization-singular states in laser images of BP layers.

\begin{tabular}{|c|c|c|c|c|c|c|}
\hline \multirow{2}{*}{$L-; \pm C-$} & \multicolumn{2}{|c|}{ Group 1} & \multicolumn{2}{c|}{ Group 2 } & \multicolumn{2}{c|}{ Group 3 } \\
\cline { 2 - 7 } & $L-$ & $\pm C-$ & $L-$ & $\pm C-$ & $L-$ & $\pm C-$ \\
\hline$Z_{1}$ & $0.71 \pm 0.079$ & - & $0.43 \pm 0.045$ & $0.14 \pm 0.032$ & $0.25 \pm 0.035$ & $0.21 \pm 0.041$ \\
\hline$Z_{2}$ & $0.12 \pm 0.034$ & - & $0.23 \pm 0.042$ & $0.39 \pm 0.091$ & $0.32 \pm 0.045$ & $0.26 \pm 0.034$ \\
\hline$Z_{3}$ & $0.05 \pm 0.008$ & - & $0.07 \pm 0.009$ & $0.41 \pm 0.12$ & $0.05 \pm 0.007$ & $0.08 \pm 0.01$ \\
\hline$Z_{4}$ & $0.17 \pm 0.044$ & - & $0.08 \pm 0.009$ & $0.49 \pm 0.23$ & $0.08 \pm 0.03$ & $0.07 \pm 0.05$ \\
\hline$S$ & $0.27 \pm 0.012$ & - & $0.24 \pm 0.02$ & $0.18 \pm 0.045$ & $0.21 \pm 0.032$ & $0.26 \pm 0.028$ \\
\hline$Q$ & $0.08 \pm 0.01$ & - & $0.01 \pm 0.0149$ & $1.01 \pm 0.35$ & $0.18 \pm 0.034$ & $0.14 \pm 0.019$ \\
\hline$F$ & $2.01 \pm 0.11$ & - & $2.12 \pm 0.012$ & - & $2.23 \pm 0.08$ & $2.05 \pm 0.11$ \\
\hline$D$ & $0.25 \pm 0.073$ & - & $0.27 \pm 0.012$ & $0.49 \pm 0.021$ & $0.22 \pm 0.019$ & $0.25 \pm 0.021$ \\
\hline
\end{tabular}

The possibility to differentiate "group" optical properties of all groups BP layers is illustrated in Table 2 .

Table 2. The efficiency of BP laser images singular analysis during the diagnostics and differentiation of breast pathological states.

\begin{tabular}{|c|c|c|c|c|}
\hline BP layers & \multicolumn{2}{|c|}{ Groups $1-2 ; 3$} & \multicolumn{2}{c|}{ Groups 2-3 } \\
\hline Parameters & $N_{L}$ & $N_{ \pm C}$ & $N_{L}$ & $N_{ \pm C}$ \\
\hline$Z_{1}$ & $\oplus$ & $\otimes$ & $\otimes$ & $\otimes$ \\
\hline$Z_{2}$ & $\oplus$ & $\otimes$ & $\otimes$ & $\otimes$ \\
\hline$Z_{3}$ & $\otimes$ & $\oplus$ & $\otimes$ & $\otimes$ \\
\hline$Z_{4}$ & $\otimes$ & $\oplus$ & $\oplus$ & $\oplus$ \\
\hline$S$ & $\otimes$ & $\otimes$ & $\otimes$ & $\otimes$ \\
\hline$Q$ & $\otimes$ & $\oplus$ & $\oplus$ & $\oplus$ \\
\hline$F$ & $\otimes$ & $\oplus$ & $\otimes$ & $\otimes$ \\
\hline$D$ & $\otimes$ & $\oplus$ & $\otimes$ & $\oplus$ \\
\hline
\end{tabular}

Note: $\otimes$ - here differentiation is impossible; $\oplus$ - possible.

\section{CONCLUSIONS}

1. Analyzed in this work are the main physical mechanisms providing formation of polarization singularities in laser images of BP layers.

2. Offered are statistical, correlation and fractal parameters for polarization-singular estimating the optical properties inherent to BP layers of all types.

3. Determined are the ranges for changing the set of criteria that characterize distributions of the amount of polarization-singular states in laser images, which enabled us to realize as diagnostics as differentiation of breast pathological changes.

\section{REFERENCES}

[1] de Boer, J.F. and Milner, T.E., "Review of polarization sensitive optical coherence tomography and Stokes vector determination," J. Biomed. Opt. 7, 359-371 (2002).

[2] de Boer, J.F., Milner, T.E. and Nelson, J.S., [Trends in Optics and Photonics (TOPS): Advances in Optical Imaging and Photon Migration], Washington, OSA (1998).

[3] Everett, M.J., Shoenenberger, K., Colston, B.W. and da Silva, L.B., "Birefringence characterization of biological tissue by use of optical coherence tomography," Opt. Lett. 23, 228-230 (1998).

[4] Shuliang, J., Wurong, Yu., Stoica, G. and Lihong, V., "Optical fiber based Mueller optical coherence tomography," Opt. Lett. 28, 1206-1208 (2003). 
[5] Angelsky, O.V., Hanson, S.G., Maksimyak, A.P., Maksimyak, P.P., "On the feasibility for determining the amplitude zeroes in polychromatic fields," Opt. Express 13, 4396-4405 (2005).

[6] Angelsky, O.V., Besaha, R.N., Mokhun, I.I., "Appearance of wave front dislocations under interference among beams with simple wave fronts," Optica Applicata 27, 272-278 (1997).

[7] Angelsky, O.V., Burkovets, D.N., Maksimyak, P.P., Hanson, S.G., "Applicability of the singular optics concept for diagnostics of random and fractal rough surfaces," Appl. Opt. 42, 4529-4540 (2003).

[8] Angelsky, O.V., Maksimyak, P.P., Hanson, S.G., Ryukhin, V.V. "New Feasibilities for Characterizing Rough Surfaces by Optical-Correlation Techniques," Appl. Opt. 40, 5693-5707 (2001). 